\title{
Automatic Tachycardia Recognition
}

\author{
ROBERT ARZBAECHER, THOMAS BUMP, JANICE JENKINS, KATHERINE \\ GLICK, FRAN MUNKENBECK, JEFFREY BROWN, and \\ N. NANDHAKUMAR \\ From the Pritzker Institute of Medical Engineering, IIT Center, and Pritzker School of Medicine, \\ University of Chicago, Chicago, Illinois, and the University of Michigan, Ann Arbor, Michigan
}

\begin{abstract}
ARZBAECHER, R., ET AL.: Automatic tachycardia recognition. A microcomputer algorithm for tachycardia identification, suitable for use in an implanted antitachycardia pacemaker, is described. The system employs an atrial and ventricular electrogram, detects a sustained fast rate in either chamber, and awakens the main program to perform detailed analysis of the tachycardia and its immediately preceding beats. The algorithm distinguishes atrial, ventricular, and AV nodal and re-entrant tachycardia from high rates due to sinus tachycardia. For testing of the program, we used a data base of twenty-two tape-recorded and documented arrhythmias provoked during electrophysiologic studies in which atrial and ventricular bipolar electrodes were in place; twenty-one of twenty-two were successfully detected. These included atrial fibrillation, atrial flutter, atrial tachycardia, AV nodal re-entrant tachycardia, AV re-entrant tachycardia using an accessory pathway, and ventricular tachycardia with and without ventriculo-atrial conduction. (PACE, Vol. 7, May-June, Part II, 1984)
\end{abstract}

antitachycardia, microcomputer, algorithm, extrastimulus testing, automatic

\section{Introduction}

Antitachycardia pacemakers presently undergoing clinical study (Cordis* ORTHOCOR II, Intermedics ** CYBERTACH, Medtronict SYMBIOS, Siemens $\neq$ TACHYLOG, Telectronics ${ }^{\circ}$ PASAR) detect tachycardia by sensing a high rate in the chamber to be paced. The specific criteria to be met before pace-termination is attempted are similar in all of these devices. The rate must be above a pre-set threshold and the tachycardia must persist for a pre-selected number of beats. These simple criteria may be insufficient for accurate arrhythmia identification and may result in inappropriate pacing and a subsequent acceleration or even initiation of the tachycardia. Camm et al., ${ }^{1}$ Bexton et al., ${ }^{2}$ and Furman et al. ${ }^{3}$ have suggested

\footnotetext{
${ }^{\star}$ Cordis Corp., Miami, Florida, U.S.A.

${ }^{\star *}$ Intermedics, Inc., Freeport, Texas, U.S.A.

+Medtronic Inc., Minneapolis, Minnesota, U.S.A.

‡Siemens-Elema, Solna, Sweden.

'Telectronics, Englewood, Colorado, U.S.A.

Address for reprints: Robert Arzbaecher, Ph.D., Director, Pritzker Institute of Medical Engineering, IIT Center, Chicago, Illinois 60616 U.S.A.
}

general schemes for incorporating other criteria. We are also of the opinion that improved specificity could be gained if the algorithm were implemented in a computer instead of in analog and digital hardwired circuits, and if the decision rules were expanded to utilize information about the activation of both atrium and ventricle.

At this time there are no commercially available computer arrhythmia diagnosis systems that incorporate atrial activation into the diagnostic logic of arrhythmia monitoring. The reason for this has been the difficulty of automatic P-wave identification in surface leads used for coronary care or Holter monitoring. We had addressed this problem earlier ${ }^{4}$ by using an easily swallowed pill electrode ${ }^{5}$ with which esophageal recording shows $\mathrm{P}$-waves that are three-to-five times larger than the QRS. This allows automatic recognition of atrial activation even when it is simultaneous with ventricular activity. For sensing in an antitachycardia pacemaker we have replaced our esophageal and surface leads with atrial and ventricular endocardial leads, respectively, but have retained the basic structure of our earlier program in which all intervals between successive depolarizations are measured and scanned for arrhythmia patterns. 
This paper describes the system we have developed for identification of a wide assortment of tachycardias based on computer analysis of electrograms from both chambers. We believe that modern CMOS integrated circuit technology makes implantation of such a system quite feasible: microprocessor chips are now available which are small enough in size and battery consumption to be included in a modern pacemaker, and which still provide enough memory and computational power for reasonably specific tachycardia diagnosis.

\section{Methods}

\section{Computer System}

Our computer algorithm follows the overall scheme of Figure 1. To conserve power, the microprocessor remains in a standby mode except for 4-millisecond periods when an atrial (A) or ventricular (V) event is sensed, during which the intervals AA and VA or VV and AV, respectively, are computed, tested for a sustained high rate and stored in a 36-event memory. A patient with a heart rate of 72 beats per minute has 2.4 events (A and V) per second, and if each awakens the microprocessor for $4 \mathrm{~ms}$, the computer is running less than $10 \mathrm{~ms}$ per second resulting in a duty cycle of less than $1 \%$ - a very light battery drain. It is only when a run of short intervals is detected that the main diagnostic program is called and full computer analysis of the most recent 36 events occurs.

Figure 2 is a flow chart of the main diagnostic program which is invoked when a sustained run of short intervals is detected in either chamber. This program first determines if the events in the tachycardia are predominantly atrial or ventricular. If atrial, the rate is computed and a diagnosis is made of atrial fibrillation (greater than 330 per minute), atrial flutter (between 240 and 330 per minute), or atrial tachycardia with second degree atrioventricular block. On the other hand, if there are more ventricular than atrial events in the run, a diagnosis is made of ventricular tachycardia.

The left and right branches of the flow chart of Figure 2 are the relatively straight-forward routes to diagnosis of a tachycardia. A more difficult task is the identification of $1: 1$ tachycardia, in which

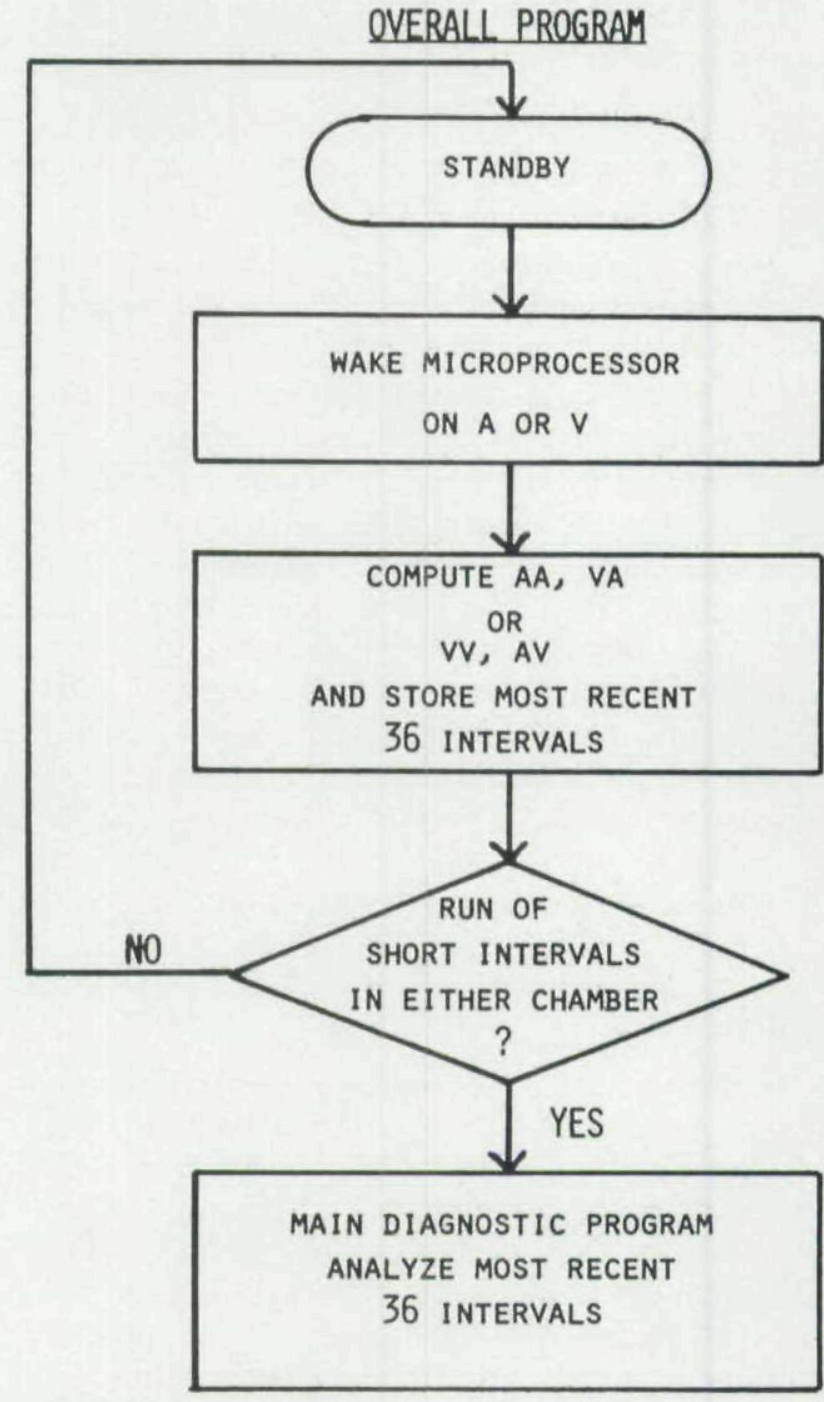

Figure 1. Flow chart of the tachycardia detection program. Ninety-nine percent of the time is spent in lowpower, standby mode, until a sustained tachycardia is detected.

the computer memory shows approximately the same number of atrial and ventricular events. Here it is important to rule out sinus tachycardia, and the program does this by studying the immediately preceding beats to determine whether the onset of the tachycardia was gradual or sudden, as has been suggested by Camm and Ward. ${ }^{6}$ In particular, the first four beats that satisfy the high rate condition that invoked the program are compared to the four beats which preceded them. If any has an interbeat interval $25 \%$ shorter than the average of the 


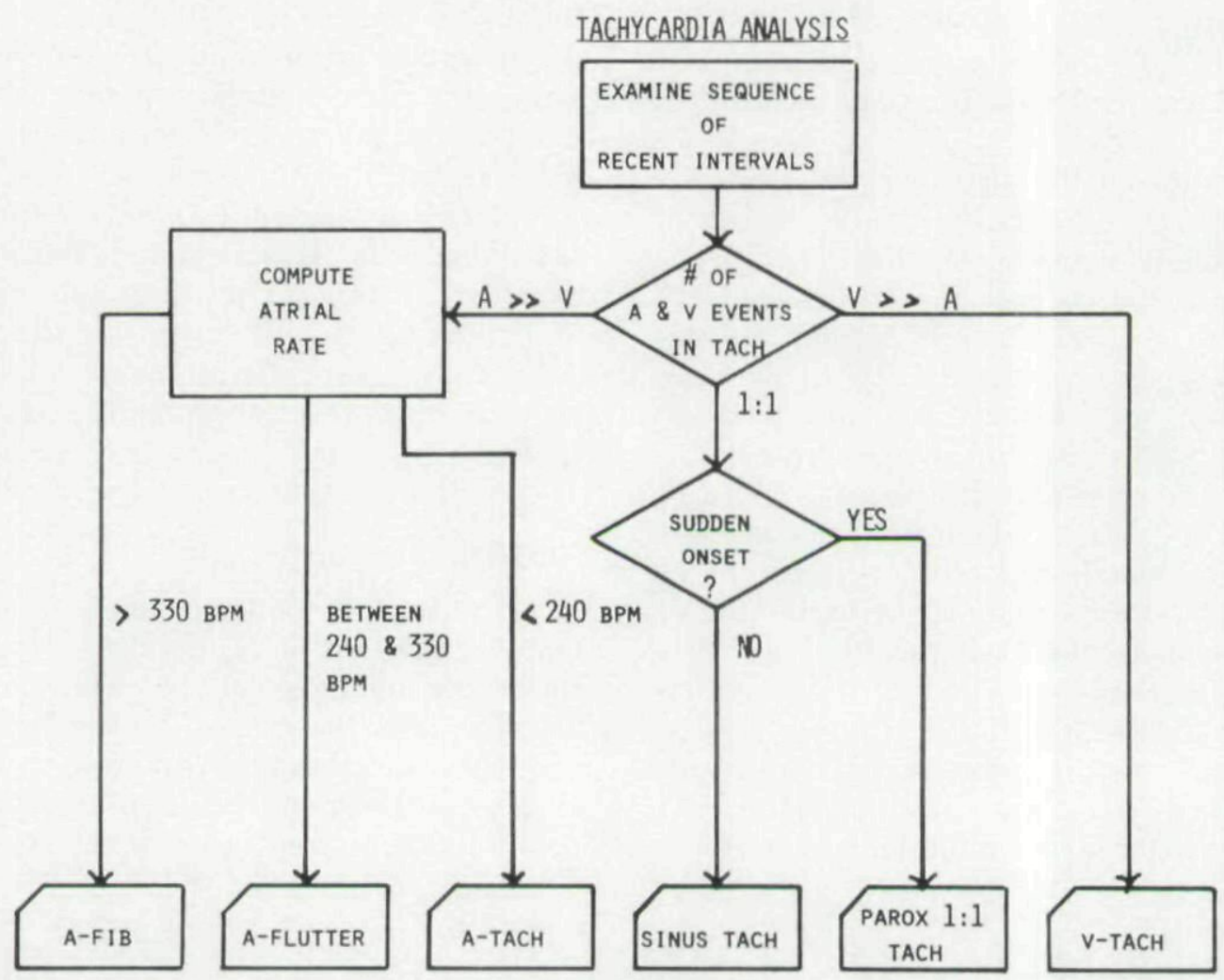

Figure 2. Flow chart of the main diagnostic program. Dual-chamber sensing allows separation of atrial and ventricular tachycardia, and the analysis of onset distinguishes sinus tachycardia.

preceding four, the tachycardia is paroxysmal and, hopefully, pace-terminable. Otherwise, the diagnosis is sinus tachycardia.

Sinus tachycardia resulting from fright, excitement, sudden effort, or postural change can occasionally develop very quickly, while so-called paroxysmal tachycardias may begin quite hesitantly, with couplets and triplets separated by long pauses. Thus, suddenness of onset will not always be a suitable characteristic for distinguishing paceterminable and non-pace-terminable tachycardia. We have developed a totally new concept, which appears to hold promise for providing this distinction, in which a premature atrial stimulus is delivered during a 1:1 tachycardia. If the tachycardia is of sinus origin, the ventricular response will be similarly premature. On the other hand, if the tachycardia is AV re-entrant or ventricular with VA conduction, the atrial premature depolarization will reach the AV node when that structure is refractory and thus will not propagate to the ventricle and perturb the tachycardia. In the implementation of this concept it is necessary for the computer program to measure carefully the rate and regularity of a tachycardia, interpose a latediastolic premature atrial beat and observe the resulting effect on the subsequent ventricular event. The tachycardia is of sinus origin if and only if the time of arrival of the next ventricular beat is disturbed by the extra stimulus.

\section{Patients}

Development of the system was based on 35 taperecordings of arrhythmias in 26 patients from an earlier study ${ }^{4}$ in which the pill electrode was used to obtain the atrial electrogram and the surface Rwave was used to simulate the ventricular electrogram. The algorithm was implemented on a microcomputer (Intel 8085) with $16 \mathrm{~K}$ of random access memory and a single $\mathrm{I} / \mathrm{O}$ port and external clock. We have not yet attempted miniaturization 
for implantation, but the program is designed to operate on-line in real time, even though our input signals at present are derived from tape recordings, not patients.

For evaluation of the algorithm, we have selected twenty-two electrically induced tachycardias from patients whose electrograms were taperecorded during intracardiac electrophysiologic study. Fifteen of these recordings (from ten patients) were given to us by Prof. Hein Wellens, University of Limburg at Maastricht; the others are recent recordings made during our own electrophysiology studies at The University of Chicago. All recordings contain at least three surface and four bipolar catheter leads, including electrograms from the right atrium, right ventricle, bundle of His and coronary sinus. Only the atrial and ventricular electrograms were used by the computer, but all were available to the electrophysiologist, who independently diagnosed each recording for comparison with the computer diagnosis.

In addition to these recordings for program eval- uation, ten patients were studied at The University of Chicago to evaluate our proposed concept for automatic atrial extrastimulus testing during tachycardia. Six of these ten were tested during sinus tachycardia (induced by atropine and isoproterenol) and seven during their pace-terminable tachycardia. After induction and stabilization of the tachycardia, atrial extrastimuli were applied at various coupling intervals and the ventricular response was carefully measured from $100 \mathrm{~mm} / \mathrm{s}$ strip-chart recordings. AV conduction curves were drawn.

\section{Results}

Figures 3, 4, and 5 are examples of three of the tachycardias used in testing the program. In each figure, the top trace, not used by the computer, is a surface lead; the second and third are atrial and ventricular electrograms, respectively. Twenty-two such records were processed automatically by the computer and twenty-one were correctly diag-

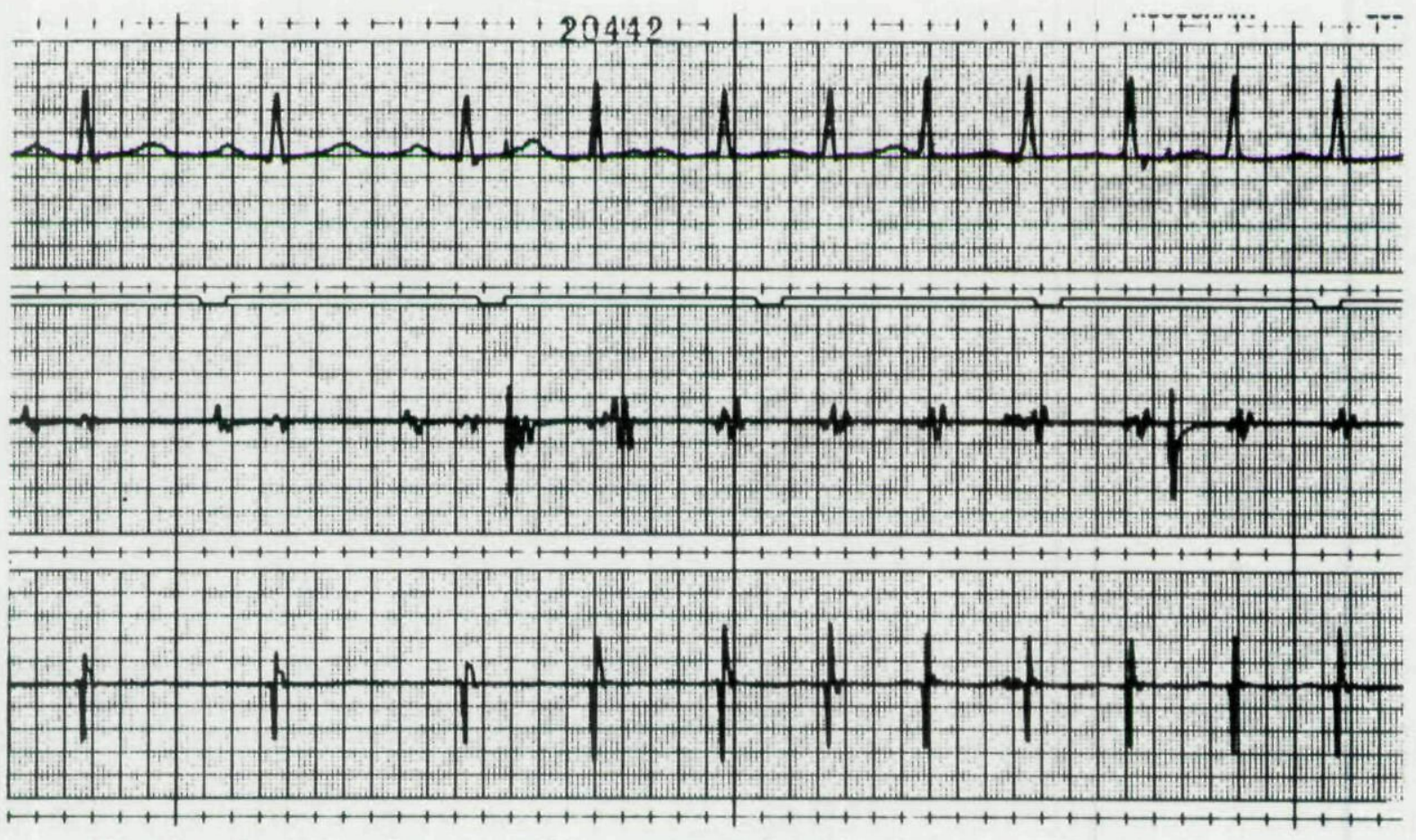

Figure 3. Initiation of an AV nodal re-entrant tachycardia that was analyzed by the microcomputer program. In this and the next two figures the top trace is a surface electrocardiogram, shown for explication of the arrhythmia but not used by the computer for analysis. The second and third traces are atrial and ventricular bipolar recordings, respectively. 


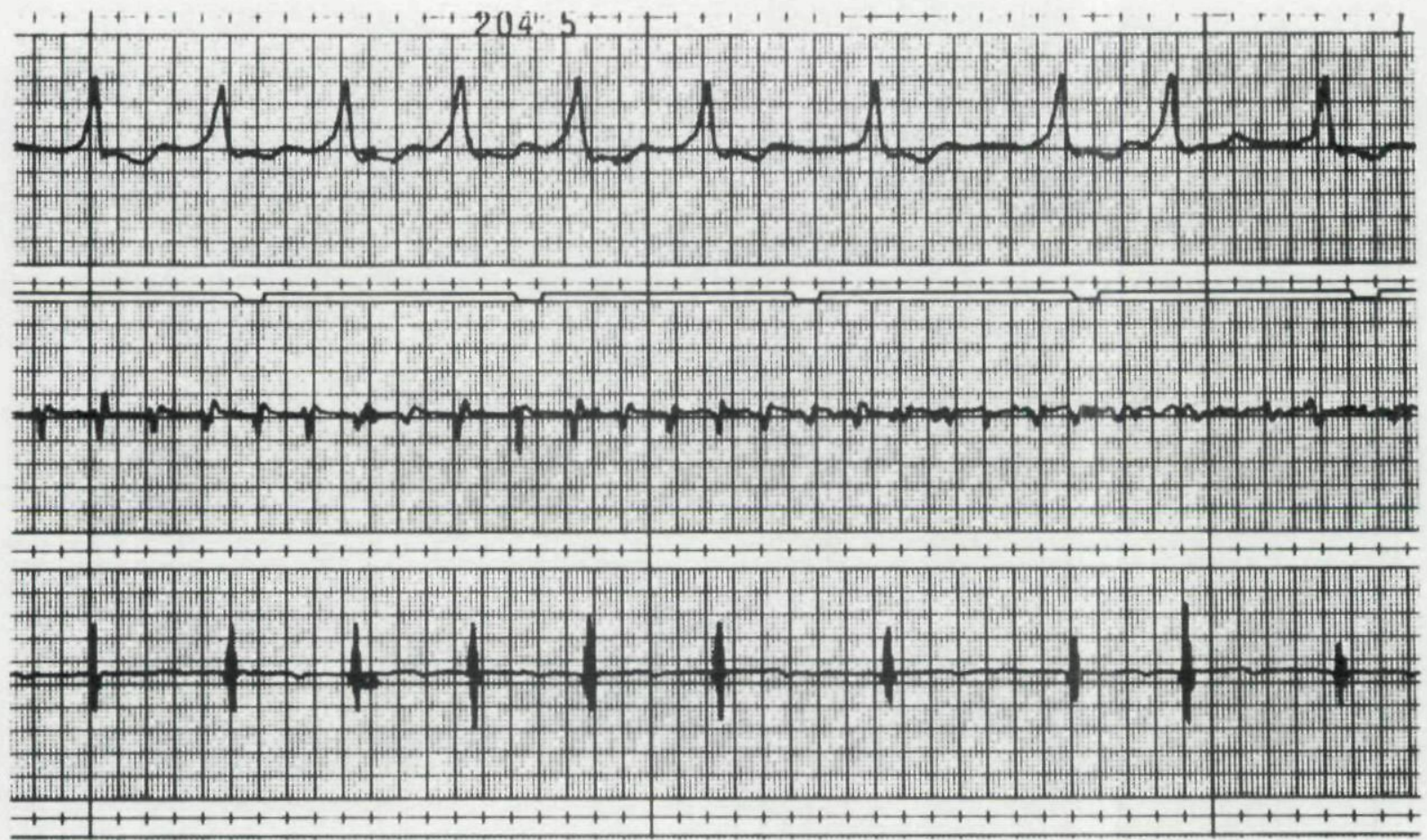

Figure 4. Development of atrial fibrillation, which was detected and classified by the microcomputer program.

nosed. These included: atrial fibrillation (2), atrial flutter (2), atrial tachycardia (2), ventricular tachycardia (4), 1:1 paroxysmal tachycardia (9), and sinus tachycardia (3). The single error occurred during an "almost 1:1" ventricular tachycardia which conducted in a retrograde direction to the atria on seven of eight beats. The electrophysiologist noted the single occurrence of ventriculoatrial block and made the diagnosis of ventricular tachycardia, while the computer reported $1: 1$ tachycardia. In addition to the tachycardias which awakened the program and elicited a diagnosis, there were many recorded episodes of non-sustained tachycardias which the computer correctly ignored.

Our idea for mid-tachycardia delivery of atrial premature stimuli to shorten (sinus tachycardia) or not shorten (other tachycardias) the subsequent ventricular interval has not yet been incorporated into the program, but has been tested successfully in nine patients undergoing electrophysiology studies for the selection of suitable antiarrhythmic drugs. With an atrial prematurity of $80 \mathrm{~ms}$, the resulting ventricular prematurity was more than $30 \mathrm{~ms}$ in 7 of 7 patients when tested during ectopic tachycardia, but less than $10 \mathrm{~ms}$ in 6 of 6 patients when tested during sinus tachycardia.

\section{Discussion}

Dual-chamber sensing provides a clear distinction among general types of tachycardia, and an even finer distinction can be made between atrial tachycardia, flutter, and fibrillation on the basis of rate. In some cases there may exist a rate overlap between atrial fibrillation and atrial flutter. In this range of heart rates it might be more appropriate to use regularity of rate as the hallmark of atrial flutter, ${ }^{7}$ but we have not yet implemented this idea. Similarly, loss of atrial sensing during atrial fibrillation was not present in our limited group of patients, but is likely to represent a problem in a larger population. We intend to incorporate an additional rule in which ventricular irregularity is the clue to the diagnosis of atrial fibrillation when distinct atrial complexes are absent. 


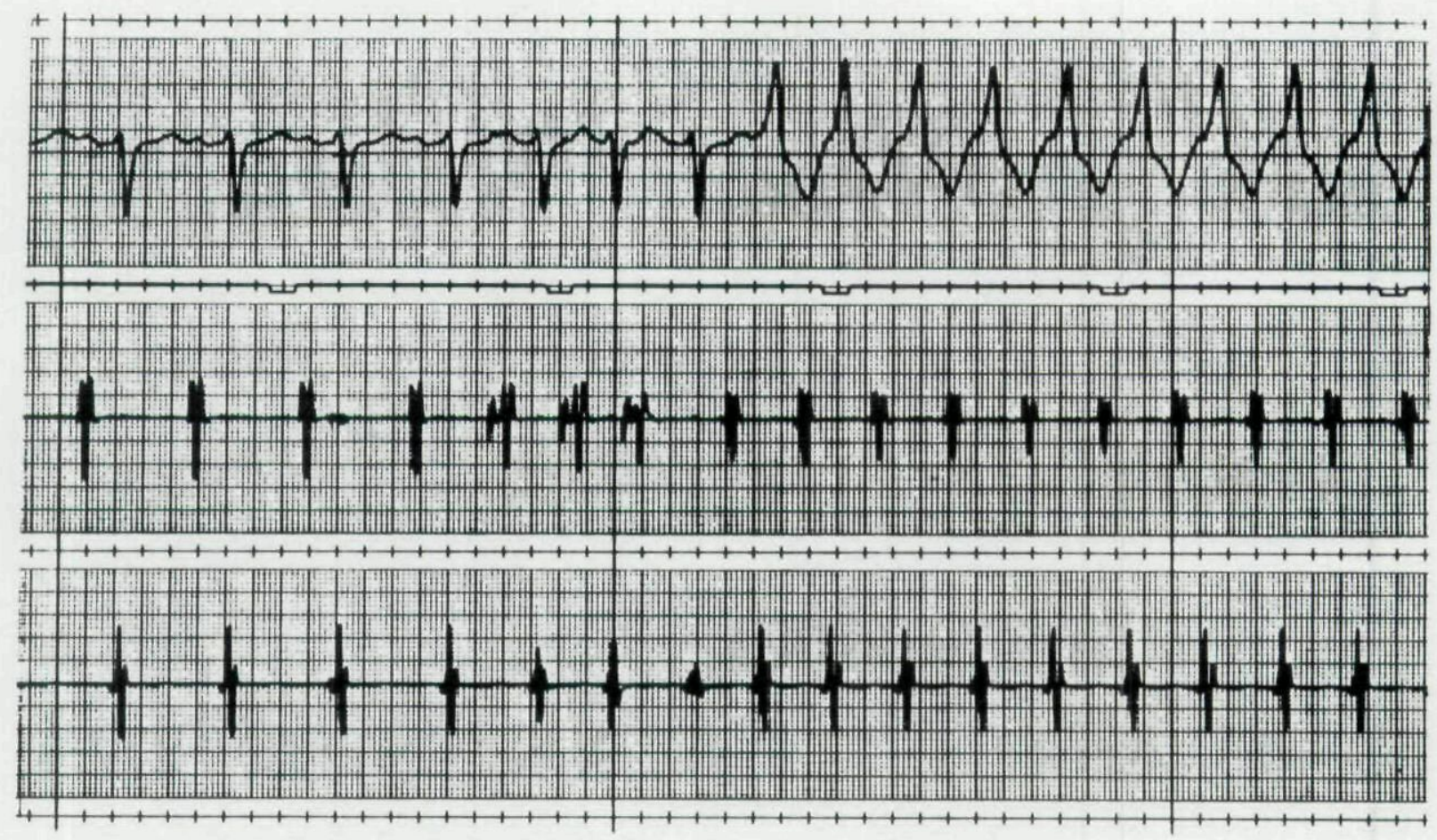

Figure 5. Onset of an AV re-entrant tachycardia, with aberrant conduction, which was detected and correctly classified by the microcomputer program as paroxysmal 1:1 tachycardia.

Tachycardias with a 1:1 AV relationship are difficult to divide further. The very important case of sinus tachycardia is identified in our program by determining suddenness of onset, which may not be a completely sensitive and specific criterion for separating sinus from pace-terminable 1:1 tachycardias. Particularly in the case of ventricular tachycardia (which frequently exhibits $1: 1$ behavior due to retrograde ventriculo-atrial conduction), it is common for the initiation to be a mixture of premature beats and pauses. As a result, the average heart rate at the beginning of such a tachycardia may not be dramatically greater than that during its prodrome, unless rather long passages are averaged. Such averaging must be done very carefully. In sinus tachycardia due to sudden exertion or excitement, comparing the average rate of the four preceding beats with that of those following the arrival at a pre-set rate may produce a difference as great as that seen in some true paroxysmal ventricular tachycardias.
Because of these uncertainties, we have designed, but not yet programmed, an extra stimulus test for distinguishing sinus tachycardia from paceterminable 1:1 tachycardia; we have preliminary evidence that the idea is sound and should be added to our sudden-onset criteria. A third method of distinguishing sinus tachycardia from tachycardias originating in lower structures is to discriminate between antegrade and retrograde activation of the atria by noting changes in the shape, amplitude, and slew rate of the atrial electrogram associated with retrograde conduction. ${ }^{8}$ As electrode fixation and electrogram stability improve in the future, this idea may become quite important not only for identifying sinus tachycardia but also for separating individual types of paroxysmal $1: 1$ tachycardias by their distinctive waveforms.

Acknowledgment: We are grateful to Professor Hein J.J. Wellens, University of Limburg, for providing us with tape-recorded tachycardia examples with which to test our algorithm. 


\section{References}

1. Camm, A.J., Nathan, A.W., and Ward, D.E.: Responsive pacing for tachycardia termination. Vectors, 9:2, 1981.

2. Bexton, R., Ward, D.E., Hellestrand, K., et al.: QT interval during paroxysmal junctional tachycardia. Eur. Heart J., 2:186, 1981.

3. Furman, S., Fisher, J.D., and Pannizo, F.: Necessity of signal processing in tachycardia detection. In S.S. Barold and J. Mugica (Eds.): The Third Decade of Cardiac Pacing, New York, Futura Publishing Company, 1982, p. 265.

4. Jenkins, J., Wu, D., and Arzbaecher, R.: Computer diagnosis of supraventricular and ventricular ar- rhythmias: a new esophageal technique. Circulation, 60:977, 1979.

5. Arzbaecher, R.: A pill-electrode for the study of cardiac arrhythmias. Med. Instrum., 12:277, 1978.

6. Camm, A.J. and Ward, D.E.: Pacing for Tachycardia Control. Telectronics, 1983, p. 139.

7. Wells, J.L. Jr., MacLean, W.A.H., James, T.N., et al.: Characterization of atrial flutter: Studies in man after open heart surgery using fixed atrial electrodes. Circulation, 60:665, 1979 .

8. Amikam, S. and Furman, S.: A comparison of antegrade and retrograde atrial depolarization in the electrogram. PACE, 6:310, 1983. 
Copyright of Pacing \& Clinical Electrophysiology is the property of Blackwell Publishing Limited and its content may not be copied or emailed to multiple sites or posted to a listserv without the copyright holder's express written permission. However, users may print, download, or email articles for individual use. 